\title{
ORTHOCENTRIC PROPERTIES OF THE PLANE $n$-LINE*
}

BY

\section{F. MORLEY}

In continuation of a memoir in these Transactions (vol. 1, p. 97) I consider the problem :

To find for $n$ lines of a plane natural metrical analogues of the elementary facts that the perpendiculars of 3 lines meet at a point (the orthocenter of the 3-line) and that the orthocenters of the 3-lines contained in a 4-line lie on a line.

I apply first to the special case of a 4-line the treatment sketched in $\S 7$ of the memoir cited; this affords suggestions for the general case.

\section{$\S 1$. The Deltoid.}

To discuss with the minimum of trouble the metrical theory of a 4-line we should take, according to our purpose, the lines as tangents either of a parabola or of a hypocycloid of class three. We want here the latter curve. As it is to most geometers an incidental stationary thing and not a weapon, I will treat it $a b$ initio. And as it is at least as good as other curves which have a given name I will call it a deltoid. It is hardly necessary to remark that it is the metrically normal form of the general rational plane curve of class three with isolated double line.

Denote by $t$ a turn or a complex number of absolute value 1 ; and think of $t$ as a point on the unit circle. We consider three points $t_{1}, t_{2}, t_{3}$ on the circle subject to the condition

$$
s_{3} \equiv t_{1} t_{2} t_{3}=1 \text {. }
$$

With this triad we associate a point $x$ by the equation

$$
x=t_{1}+t_{2}+t_{3} \equiv s_{1},
$$

which carries with it the conjugate equation

$$
y=1 / t_{1}+1 / t_{2}+1 / t_{3}=t_{2} t_{3}+t_{3} t_{1}+t_{1} t_{2} \equiv s_{2} .
$$

We have from (1) and (2)

$$
x=t_{1}+t_{2}+1 / t_{1} t_{2} .
$$

* Presented to the Society at the Evanston meeting, September 2-3, 1902. Received for publication September 3, 1902. 
Herein let $t_{1}$ be fixed, $t_{2}$ variable. Since $x=t+1 / t$ is that simplest of all hypocycloids, the segment of a line, so also is (3) the segment of a line, of constant length 4 , with center $t_{1}$ and ends where

or

$$
D_{t_{2}} x=0 \text {, }
$$

$$
t_{1} t_{2}^{2}=1
$$

or

$$
x=t_{1} \pm 2 / \sqrt{t_{1}} \text {. }
$$

When $t_{1}$ varies, the deltoid is described by the motion of this variable segment ; the ends move on the curve, and the segment touches the curve. And any point inside the curve is given by (3) as the intersection of 2 segments; the points of the curve itself are given when the segments are brought to coincidence, that is, are

$$
x=2 t+1 / t^{2} \text {. }
$$

This equation expresses that while a point $2 t$ is describing a circle of radius 2 another point $x$ moves round it with an angular velocity opposite in sense and twice as great; thus the cycloidal nature of the curve is apparent.

A point of the curve and also a line of the curve is named by its parameter $t$; thus the point $t$ of the curve is given by (4), and the line $t$ from (1) and (2) by

$$
x t^{2}-y t=t^{3}-1 \text {. }
$$

$x$ and $y$ being always conjugate coördinates.

Now two lines $t_{1}$ and $t_{2}$ meet at

$$
x_{1}=t_{1}+t_{2}+1 / t_{1} t_{2} .
$$

Symmetrize this equation for 3 lines of the curve by writing it

$$
x=s_{1}-t_{3}+t_{3} / s_{3} \text {. }
$$

Omitting the suffix of $t_{3}$ we have the map-equation of the circumcircle. Hence : The circumcenter of the 3 -line is $s_{1}$, and the circumradius is $\left|1-1 / s_{3}\right|$ or $\left|1-s_{3}\right|$.

The mean point of

is

$$
t_{1}+t_{2}+1 / t_{1} t_{2} \text { and } t_{1}+t_{3}+1 / t_{1} t_{3}
$$

$$
m=\frac{1}{2}\left[s_{1}+t_{1}+\left(s_{1}-t_{1}\right) / s_{3}\right] \text {. }
$$

Hence the center of the circle which bisects the sides, the Feuerbach or ninepoint circle, is

$$
c=\frac{1}{2}\left(s_{1}+s_{1} / s_{3}\right) .
$$


The perpendicular from $t_{1}+t_{2}+1 / t_{1} t_{2}$ on the line $t_{3}$ is

$$
x t_{3}+y=t_{3}\left(t_{1}+t_{2}+1 / t_{1} t_{2}\right)+1 / t_{1}+1 / t_{2}+t_{1} t_{2}=s_{2}+t_{3} s_{1} / s_{3} .
$$

Hence the orthocenter is

$$
p=s_{1} / s_{3},
$$

as could of course be inferred from the fact that

$$
\text { orthocenter }+ \text { circumcenter }=2 \times \text { Feuerbach center } .
$$

Since then $|p|=\left|s_{1}\right|$ we have:

Theorem 1. The center of a deltoid, of which three lines are given, is equidistant from the circumcenter and orthocenter of the 3-line.

The centre of the deltoid which touches 4 lines is thus determined as the intersection of 4 lines, one for each of 3 of the given 4 .

The Feuerbach center for 3 of 4 lines is given by

$$
2 c=\left(s_{1}-t_{4}\right)\left(1+t_{4} / s_{4}\right), \quad(s \text { for } 4),
$$

$s_{1}$ and $s_{3}$ being replaced in (7) by symmetric functions of $t_{1}, t_{2}, t_{3}, t_{4}$. Thus the 4 such centers are included in

whose conjugate is

$$
2 x=\left(s_{1}-t\right)\left(1+t / s_{4}\right),
$$

But

Therefore

$$
2 y=\left(s_{3} / s_{4}-1 / t\right)\left(1+s_{4} / t\right)=\left(s_{3} / t-s_{4} / t^{2}\right)\left(1+t / s_{4}\right) .
$$

$$
t^{2}-s_{1} t+s_{2}-s_{3} / t+s_{4} / t^{2}=0 \text {. }
$$

$$
2(t x+y)=s_{2}\left(1+t / s_{4}\right),
$$

a line through $\frac{1}{2} s_{2} / s_{4}$, perpendicular to the line $t$ of the curve. Hence

Theonem 2. If from the Feuerbach center of any 3 of 4 lines a perpendicular be drawn to the remaining line, the 4 perpendiculars meet at a point, namely $\frac{1}{2} s_{2} / s_{4}$.

\section{§2. Extension to the n-line.}

I employ now the notation of $\S 2$ of the memoir cited, namely I write a line $l_{a}$ in the form

$$
x t_{\alpha}+y=x_{a} t_{\alpha}=y_{\alpha} .
$$

Denote the characteristic constants by $a_{a}$,

$$
a_{a}=\sum \frac{x_{1} t_{1}^{n-a}}{\left(t_{1}-t_{2}\right) \cdots\left(t_{1}-t_{n}\right)}, \quad(a=1,2, \cdots, n)
$$


and their conjugates by $b_{a}$, so that

$$
b_{\alpha}=(-)^{n-1} s_{n} a_{n+1-\alpha}
$$

where $s_{n}=t_{1} t_{2} \cdots t_{n}$, and in general $s_{a}=\Sigma t_{1} t_{2} \cdots t_{a}$ for $n t^{\prime}$ s.

The circumcenter of a 3-line is $a_{1}$. The mean point of the joins of $l_{1}, l_{2}$ and $l_{1}, l_{3}$ is given by

$$
\begin{aligned}
2 m & =\frac{x_{1} t_{1}}{t_{1}-t_{2}}+\frac{x_{2} t_{2}}{t_{2}-t_{1}}+\frac{x_{1} t_{1}}{t_{1}-t_{3}}+\frac{x_{3} t_{3}}{t_{3}-t_{1}} \\
& =\frac{x_{1} t_{1}\left(2 t_{1}-s_{1}+t_{1}\right)}{\left(t_{1}-t_{2}\right)\left(t_{1}-t_{3}\right)}+\frac{x_{2} t_{2}\left(2 t_{2}-s_{1}+t_{1}\right)}{\left(t_{2}-t_{1}\right)\left(t_{2}-t_{3}\right)}+\frac{x_{3} t_{3}\left(2 t_{3}-s_{1}+t_{1}\right)}{\left(t_{3}-t_{1}\right)\left(t_{3}-t_{2}\right)},
\end{aligned}
$$

and therefore the nine-point circle is

and its center is

$$
2 m=2 a_{1}-s_{1} a_{2}+a_{2} t,
$$

Hence the orthocenter is

$$
2 c=2 a_{1}-s_{1} a_{2} \text {. }
$$

$$
p=2 c-a_{1}=a_{1}-s_{1} a_{2} \text {. }
$$

The line about the points $a_{1}$ and $p$ (which is the locus of centers of inscribed deltoids) is, when $\tau$ is any turn,

$$
x-a_{1}+s_{1} a_{2}=\left(x-a_{1}\right) \tau .
$$

This for 3 of 4 lines is

$$
x-a_{1}+t a_{2}+\left(s_{1}-t\right)\left(a_{2}-t a_{s}\right)=\left(x-a_{1}+t a_{2}\right) \tau,
$$

or

$$
x-a_{1}+s_{1} a_{2}-t\left(s_{1}-t\right) a_{3}=\left[x-a_{1}+s_{1} a_{2}-\left(s_{1}-t\right) a_{2}\right] \tau \text {. }
$$

Now since for 4 lines $a_{3} / a_{2}$ is a turn we can equate $t a_{3}$ to $a_{2} \tau$; that is, whatever turn $t$ may be, the line passes through the point

$$
p_{1}=a_{1}-s_{1} a_{2} \text {. }
$$

This is then the center of the inscribed deltoid of the 4-line.

For 4 of 5 lines this point is given by

$$
p=a_{1}-t a_{2}-\left(s_{1}-t\right)\left(a_{2}-t a_{3}\right)=a_{1}-s_{1} a_{2}+t\left(s_{1}-t\right) a_{3},
$$

or the conjugate equation

But for 5 things

$$
q=b_{1}-\frac{s_{4}}{s_{5}} b_{2}-\frac{1}{t}\left(\frac{s_{4}}{s_{5}}-\frac{1}{t}\right) b_{3}=s_{5} a_{5}-s_{4} a_{4}+\left(\frac{s_{4}}{s_{5}}-\frac{s_{5}}{t^{2}}\right) a_{3} .
$$

$$
t^{3}-t^{2} s_{1}+t s_{2}-t s_{3}+s_{4} / t-s_{5} / t^{2}=0 .
$$


Therefore

$$
t\left(p-a_{1}+s_{1} a_{2}\right)-\left(q-s_{5} a_{5}+s_{4} a_{4}\right)=\left(t s_{2}-s_{3}\right) a_{3},
$$

that is, the line joining the $p$ of 4 lines to the point $a_{1}-s_{1} a_{2}+s_{2} a_{3}$ is perpendicular to the remaining line; or

Theonem 3. If from the center of the inscribed deltoid of 4 of 5 lines a perpendicular be drawn to the line left out, the 5 perpendiculars meet at a point ; namely the point

$$
p_{2} \equiv a_{1}-s_{1} a_{2}+s_{2} a_{3} .
$$

Call this point the first orthocenter of the 5-line.

For 5 of 6 lines this point is

$$
\begin{aligned}
x & =a_{1}-t a_{2}-\left(s_{1}-t\right)\left(a_{2}-t a_{3}\right)+\left(s_{2}-t s_{1}+t^{2}\right)\left(a_{3}-t a_{4}\right) \\
& =a_{1}-s_{1} a_{2}+s_{2} a_{3}-t\left(s_{2}-t s_{1}+t^{2}\right) a_{4} \\
& =p_{2}-t\left(s_{2}-t s_{1}+t^{2}\right) a_{4}
\end{aligned}
$$

or, if the conjugates be written,

But for 6 things

$$
y=q_{2}+\left(s_{4} / t-s_{5} / t^{2}+s_{6} / t^{3}\right) a_{3} .
$$

Hence

$$
t^{3}-t^{2} s_{1}+t s_{2}-s_{3}+s_{4} / t-s_{5} / t^{2}+s_{6} / t^{3}=0 .
$$

that is to say

$$
\frac{x-p_{2}}{a_{4}}-\frac{y-q_{2}}{a_{3}}=-s_{3}
$$

Theorem 4. The first orthocenters of the 5-lines included in a 6-line lie on a line.

The argument is clearly general, so that if the point $p_{2} \equiv a_{1}-s_{1} a_{2}+s_{2} a_{3}$ be constructed for a 6 -line, the perpendiculars from such point for 6 of 7 lines on the line left out meet at a point, and for 7 of 8 lines these points lie on a line; and so on. Briefly, we have found an orthocenter for an odd number of lines, a directrix for an even number.

\section{§3. Construction of a series of points.}

The points to which attention is thus directed belong, for a given $n$-line, to the series

$$
p_{0}=a_{1}, \quad p_{1} \equiv a_{1}-s_{1} a_{2}, \quad p_{2} \equiv a_{1}-s_{1} a_{2}+s_{2} a_{3}, \text { etc. }
$$

Their construction is merely a matter of centroids, or centers of gravity. For we regard as known in an $n$-line: 
$a_{1}$, the center of the center-circle,

$a_{1}-t_{i} a_{2}$, the $n$ such points of the $(n-1)$-lines,

$a_{1}-\left(t_{i}+t_{k}\right) a_{2}+t_{i} t_{k} a_{3}$, the $\left(\begin{array}{l}n \\ 2\end{array}\right)$ such poinis of the $(n-2)$-lines,

and taking the centroid of each set we have $a_{1}, g_{1}, g_{2} \ldots$ where

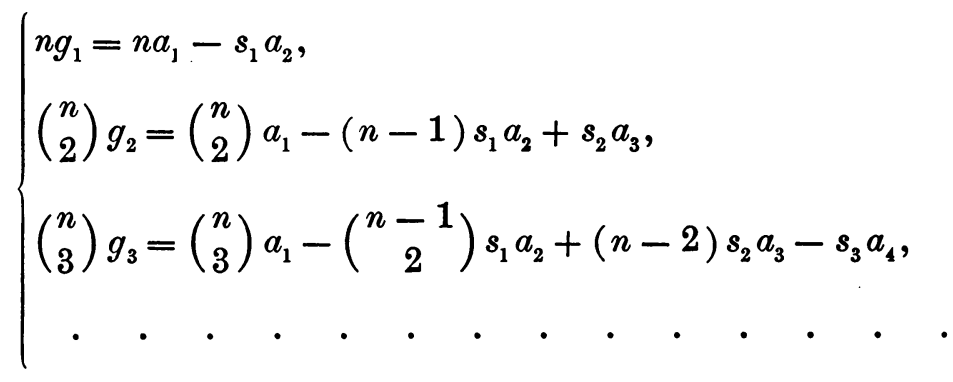

whence the $p$ 's are easily constructed. It will be noticed that the last equations cease to be independent of the origin when $a_{n}$ itself makes its appearance; thus $g_{n-1}$ is the centroid of the points $x_{i}$, the reflexions of the origin in the $n$ lines. Hence also $p_{n-1}$ is a point dependent on the origin, not a point of the $n$-line itself.

But a more vivid construction is indicated by the process by which $p_{1}$ for a 4-line was deduced (p. 4) from $a_{1}$ and $p_{1}$ for a 3-line. It will be clear on constructing $p_{1}$ for a 5-line.

We write as before (eq. 10), for a 4-line,

$$
x-p_{1}=\left(x-a_{1}\right) z,
$$

and extend this to a 5 -line, observing that $p_{1}$ for 4 is $p_{1}+t\left(s_{1}-t\right) a_{3}$ for 5 . Thus the extended equation is

$$
x-p_{1}-t\left(s_{1}-t\right) a_{3}=\left(x-a_{1}+t a_{2}\right) z=\left\{x-p_{1}-\left(s_{1}-t\right) a_{2}\right\} z .
$$

If $z a_{2}=t a_{3}$, we have $x=p_{1}$. Let then $|z|=\left|a_{3} / a_{2}\right|$. That is, if we divide the known points $p_{1}$ and $a_{1}$ of 4 of 5 lines in the ratio $\left|a_{3} / a_{2}\right|$, where the constants $a_{i}$ refer to the 5 -line, the 5 such circles meet at the point,

$$
p_{1} \equiv a_{1}-s_{1} a_{2} .
$$

And $p_{1}$ being now known for a 5 -line, we have a similar statement for a 6 -line, whence $p_{1}$ is known in general. But again we know $p_{1}$ and $p_{2}$ for a 5-line. Write for $n-1$ lines

$$
x-p_{2}=\left(x-p_{1}\right) z,
$$


and extend this. We have for an $n$-line,

$$
\begin{aligned}
x-p_{2}+t\left(s_{2}-t s_{1}+t^{2}\right) a_{4} & =\left\{x-p_{1}-t\left(s_{1}-t\right) a_{3}\right\} z \\
& =\left\{x-p_{2}+\left(s_{2}-t s_{1}+t^{2}\right) a_{3}\right\} z,
\end{aligned}
$$

whence, as before, the point $p_{2}$ of the $n$-line has its distances from the points $p_{2}$ and $p_{1}$ of any included $n-1$ lines in the fixed ratio $\left|a_{4}\right| a_{3} \mid$. And so in general :

Theorem 5. The point $p_{a}$ of an n-line has its distance from the points $p_{a}$ and $p_{a-1}$ of an included $(n-1)$-line in the fixed ratio $\left|a_{a+2} / a_{a+1}\right|$.

Since $b_{a}=(-)^{n-1} s_{n+1-a}$, this fixed ratio is unity when

or

$$
\alpha+2+\alpha+1=n+1
$$

$$
\alpha+1=\frac{1}{2} n,
$$

that is, in a $2 n$-line the point $p_{n-1}$ is equidistant from the points $p_{n-1}$ and $p_{n-2}$ of any included $(2 n-1)$-line.

Regarding the lengths $\left|a_{a}\right|$ as known, we have in Theorem 5 a construction for the points $p_{a}$ for $n$-lines, when the points $p_{a}$ for $(n-1)$-lines are known.

\section{§ 4. The curve $\Delta^{2 i-1}$.}

The peculiar appropriateness of the deltoid for the metrical theory of four lines makes it desirable to have an analogous curve for $2 n$-lines. Such a curve is

$$
\begin{aligned}
& (-)^{n}\left(x t^{n}-y t^{n-1}\right)=t^{i_{n-1}}-1-\left(s_{1} t^{2 n-2}-s_{2 n-2} t\right) \\
& +\cdots+(-)^{n}\left(s_{n-2} t^{n+1}-s_{n+1} t^{n-2}\right)
\end{aligned}
$$

where $c_{a}$ and $c_{2 n-1-a}$ are conjugate. This is a curve $\Delta^{2 n-1}$ of class $2 n-1$, order $2 n$, with a line equation of the type

$$
\xi^{n} \eta^{n}=\text { form in } \xi, \eta \text { of order } 2 n-1 .
$$

For clearness I will take the case $n=3$, next to the case $n=2$ of $\S 1$; the generalizations are immediate.

Any 6 lines are lines of a curve $\Delta^{5}$,

$$
-\left(x t^{3}-y t^{2}\right)=t^{5}-1-\left(s_{1} t^{4}-s_{4} t\right) .
$$

The map-equation of the curve is

$$
-x=3 t^{2}+2 t^{-3}-\left(2 s_{1} t-s_{4} t^{-2}\right) .
$$

Thus the curve is derived by addition from 2 concentric cycloids. 
So $\Delta^{2 n-1}$ is derived by addition from $n-1$ concentric cycloids; those points being added at which the tangents are parallel.

Let the common center of the cycloids be called the center of $\Delta^{2 n-1}$.

The cusps of $\Delta^{5}$ are given from (19) by

or

$$
6\left(t-t^{-4}\right)-2\left(s_{1}-s_{4} t^{-3}\right)=0,
$$

$$
3\left(t^{5}-1\right)=s_{1} t^{4}-s_{4} t .
$$

Hence the cusp-tangents are such that

$$
3\left(x t^{3}-y t^{2}\right)=2\left(s_{1} t^{4}-s_{4} t\right),
$$

that is :

There are 5 cusp-tangents of $\Delta^{5}$; they touch a concentric $\Delta^{3}$. And so

TheOREM 6. There are $2 n-1$ cusp-tangents of $\Delta^{2 n-1}$; they touch a concentric $\Delta^{2 n-3}$.

Consider the common lines of $\Delta^{5}$ and any $\Delta^{3}$,

$$
x t^{2}-y t=\alpha t^{3}+\beta t^{2}-\gamma t-\delta .
$$

There are 5 common lines, and they are given by

$$
t^{5}-1-\left(c_{1} t^{4}-c_{4} t\right)+t\left(\alpha t^{3}+\beta t^{2}-\gamma t-\delta\right)=0 .
$$

Hence the center of the $\Delta^{3}$ is

where

$$
\beta=\sum t_{1} t_{2}=s_{2}
$$

$$
s_{5}=1 \text {, }
$$

that is, the center of the deltoid touching 4 lines of $\Delta^{5}$ is

$$
x=s_{2}+s_{1} / s_{4} \quad(s \text { for } 4) .
$$

The perpendicular on a fifth line of $\Delta^{5}$ is

$$
x t+y=t\left(s_{2}+s_{1} / s_{4}\right)+s_{2} / s_{4}+s_{3}=s_{3}+s_{2} t / s_{5} \quad(s \text { for } 5) .
$$

Hence the first orthocenter of 5 lines is $s_{2} / s_{5}$.

For 5 of 6 lines this point is

or

$$
x=\left(t^{2}-t s_{1}+s_{2}\right) t / s_{6}
$$

$$
y=s_{6} / t^{3}-s_{5} / t^{2}+s_{4} / t
$$

whence the 6 first orthocenters lie on the line

$$
s_{6} x+y=s_{3} \text {. }
$$


The line of $\Delta^{3}$ perpendicular to a fifth line of $\Delta^{5}$ is

where

$$
x t^{2}+y t+\alpha t^{3}-\beta t^{2}-\gamma t+\delta=0,
$$

or is

$$
c_{1}-\alpha=s_{1}, \quad \beta=s_{2}, \quad \gamma=s_{3}, \quad c_{4}-\delta=s_{4}, \quad 1=s_{5} ;
$$

$x t^{2}+y t+\left(c_{1}-s_{1}-1 / s_{4}\right) t^{3}-\left(s_{2}+s_{1} / s_{4}\right) t^{2}-\left(s_{3}+s_{2} / s_{4}\right) t$

or

$$
+c_{4}-s_{4}-s_{3} / s_{4}=0 \quad(s \text { for } 4),
$$

$$
x t^{2}+y t+c_{1} t^{3}-s_{2} t^{2}-s_{4}+c_{4}-s_{3} t / s_{5}-s_{1} t^{3} / s_{5}=0 \quad(s \text { for } 5) \text {. }
$$

That is :

Theorem 7. If of the deltoid touching any 4 of 5 lines we draw the line perpendicular to the omitted line, the 5 perpendiculars touch a deltoid.

The center of this deltoid is $s_{2}$. The first orthocenter was $s_{2} / s_{5}$. These are strokes of equal size. Hence :

THeOREM 8. The locus of centers of curves $\Delta^{5}$ of. which 5 lines are given is a line. And so for $\Delta^{2 n-1}$.

The curve $\Delta^{2 n-1}$ does then for $2 n$ lines precisely what the deltoid $\Delta^{3}$ does for 4 lines; it replaces Clifford's $n$-fold parabola for metrical purposes. We have proved by its means the theorems of $\S 2$ over again with additions; in particular we have assigned a meaning to the point $p_{1}$ of a 5 -line or $p_{n-2}$ of a $(2 n-1)$-line, for this is readily identified as the point $s_{2}$ of 5 lines of $\Delta^{5}$ or $s_{n-1}$ of $2 n-1$ lines of $\Delta^{2 n-1}$. But at present I regard the use of this curve as more limited than the method of the $a$ 's, to which I now return.

\section{\$5. The second circle of an n-line.}

A curve of order $n$, whose highest terms in conjugate coördinates are

$$
t x^{n}+y^{n}
$$

has its asymptotes apolar to the absolute points $I J$, that is, these asymptotes form an equiangular polygon. Such a curve depends on $\frac{1}{2} n(n+1)+1$ constants, and therefore a pencil can be drawn through $\frac{1}{2} n(n+1)$ points in general, and the pencil determines $n^{2}-\frac{1}{2} n(n+1)$, or $\frac{1}{2} n(n-1)$ other points. In the pencil are the imaginary curves

and

the pencil itself is

$$
x^{n}+a n^{n-1}+\cdots+a^{\prime} y^{n-1}+\cdots=0
$$

$$
y^{n}+b y^{n-1}+\cdots+b^{\prime} y^{n-1} \cdots=0,
$$

$$
t n^{n}+y^{n}+\left(t a+b^{\prime}\right) x^{n-1}+\cdots=0 \text {, }
$$


the polar line of $I$ is

$$
n t x+t a+b^{\prime}=0,
$$

and therefore the polar lines of $I$ and $J$ meet on a circle.*

Let now the $\frac{1}{2} n(n+1)$ points be the joins of $n+1$ lines. We shall call the circle the second circle of the $(n+1)$-line ; the first circle being the centercircle (l. c., p. 99).

For a 3-line the second circle is the Feuerbach circle.

Now calculate the center and radius of the second circle of the $n$-line

The pencil is

$$
x t_{a}+y=x_{a} t_{a}=y_{a} \quad(\alpha=1, \cdots, n) .
$$

$$
\sum_{a} \frac{A_{i}}{n t_{\alpha}+\frac{y_{a}}{y-1}}=0
$$

The highest powers arise from

$$
\sum_{a} \frac{A_{\alpha}}{x t_{a}+y}
$$

and are to be $t x^{n-1}+y^{n-1}$, so that, if $y / x=\lambda$,

$$
t+\lambda^{n-1}=\sum A_{1}\left(t_{2}+\lambda\right)\left(t_{3}+\lambda\right) \cdots\left(t_{n}+\lambda ;\right.
$$

whence

$$
\begin{aligned}
t & =\sum A_{1} t_{2} \cdots t_{n}^{\prime} ; \\
t+\left(-t_{1}\right)^{n-1} & =A_{1}\left(t_{2}-t_{1}\right)\left(t_{3}-t_{1}\right) \cdots\left(t_{n}-t_{1}\right) .
\end{aligned}
$$

Operating with $D_{x}^{n-2}$ on

$$
\sum A_{1}\left(x t_{2}+y-y_{2}\right) \cdots\left(x t_{n}+y-y_{n}\right),
$$

we have for the polar line of $I$

or if

$$
(n-1) t x=\sum A_{1} t_{2} t_{3} \cdots t_{n}\left(x_{2}+x_{3}+\cdots+x_{n}\right),
$$

or since

$$
\sum x_{a}=n g_{n-1} \text {, }
$$

$$
(n-1) t x=n t g_{n-1}-\sum n_{1} \frac{\left\{t+\left(-t_{1}\right)^{n-1}\right\} t_{2} \cdots t_{n}}{\left(t_{2}-t_{1}\right)\left(t_{3}-t_{1}\right) \cdots\left(t_{n}-t_{1}\right)}
$$

$$
\sum x_{1} \frac{t_{2} \cdots t_{n}}{\left(t_{2}-t_{1}\right) \cdots\left(t_{n}-t_{1}\right)}=a_{1}-s_{1} a_{2}+\cdots+(-)^{n-1} s_{n-1} a_{n}=p_{n-1},
$$

the second circle is

$$
(n-1) x=n g_{n-1}-p_{n-1}-s_{n} a_{2} / t .
$$

* Cf. J. H. Grace, Proceedings London Mathematical Society, vol. 33 (1900), p. 194. 
Hence its radius is

$$
\left|\frac{s_{n} a_{2}}{n-1}\right|, \quad \text { i. e., } \quad \frac{1}{n-1}\left|a_{2}\right| \text {, or : }
$$

TheOREM 9. The radius of the second circle of an n-line is $1 /(n-1)$ of the radius of the first circle.

Also its center is given by

or explicitly by

$$
(n-1) c=n g_{n-1}-p_{n-1},
$$

$$
(n-1) c=(n-1) a_{1}-(n-2) s_{1} a_{2}+\cdots-(-)^{n} s_{n-2} a_{n-1} .
$$

Omitting now the $n$th line we have for the second center of the rest

$$
(n-2) c^{\prime}=(n-1) g_{n-2}^{\prime}-p_{n-2}^{\prime},
$$

whence

$$
(n-1) c-(n-2) c^{\prime}=x_{n}-\left(p_{n-1}-p_{n-2}^{\prime}\right) \text {. }
$$

Here

$$
\begin{aligned}
p_{n-2}^{\prime}= & a_{1}-t a_{2}-\left(s_{1}-t\right)\left(a_{2}-t a_{3}\right)+\cdots \\
& \quad+\left\{t^{n-2}-s_{1} t^{n-3}+\cdots+(-)^{n} s_{n-2}\right\}\left(a_{n-1}-t a_{n}\right) \\
= & p_{n-2}-a_{n}\left\{t^{n-1}-s_{1} t^{n-2}+\cdots+(-)^{n} t s_{n-2}\right\} \\
= & p_{n-1}-a_{n}\left\{t^{n-1}-s_{1} t^{n-2}+\cdots-(-)^{n} s_{n-1}\right\} \\
= & p_{n-1}+(-)^{n} a_{n} s_{n} / t \\
= & p_{n-1}-b_{1} / t .
\end{aligned}
$$

Therefore

$$
(n-2) c^{\prime}=(n-1) c-x_{n}+b_{1} / t_{n} .
$$

But the reflexion $r^{\prime}$ of the first center $a_{1}$ in the omitted line is $x_{n}-b_{1} / t_{n}$. Hence

$$
(n-2) c^{\prime}=(n-1) c-r^{\prime},
$$

whence it follows at once that:

TheоReм 10. If from the second center of each $(n-1)$-line of an n-line a perpendicular be drawn to the omitted line, these perpendiculars meet at a point; the point is the external center of similitude of the first and second circles of the n-line.

The point $h$ so found is given by

$$
\begin{aligned}
(n-2) h=(n-1) c-a_{1}=(n-2) & \left(a_{1}-s_{1} a_{2}\right)+(n-3) s_{2} a_{3} \\
& -(n-4) s_{3} a_{4}+\cdots(-)^{n} s_{n-2} a_{n-1} .
\end{aligned}
$$


Whereas the orthocenter of $\S 2$ applied only to an odd number of lines the present one applies to any number. We have then for an odd $n$-line two solutions of our problem, except when $n=3$, in which case the points $h$ and $p$ coincide.

But when we have two orthocenters, we have a whole line of orthocenters, since evidently a perpendicular to one of the $n$ lines, dividing the join of the two known points of the remaining $n-1$-lines in a fixed ratio, will divide the join of the two orthocenters in the same fixed ratio.

Thus we have found, for an even number of lines, one orthocenter; for an odd number of lines, a line of orthocenters; for an even number of lines, one directrix.

KNowlton, P. Q., July, 1902. 